\title{
1 Reliability surrogate measures for water distribution system 2 design: a comparative analysis
}

3 Haixing Liu ${ }^{1}$, Dragan A. Savić 2 , Zoran Kapelan ${ }^{3}$, Enrico Creaco ${ }^{4}$, Yixing Yuan ${ }^{5}$

$4{ }^{1}$ Associate Research Fellow, School of Hydraulic Engineering, Dalian University of 5 Technology, Dalian 116024, China; Formerly, State Key Laboratory of Urban Water 6 Resource and Environment, School of Municipal and Environmental Engineering, Harbin 7 Institute of Technology, Harbin, 150090, China. E-mail: lhx_526@163.com

$82^{2}$ Professor, College of Engineering, Mathematics and Physical Sciences, University of 9 Exeter, Exeter, EX4 4QF, UK. E-mail: d.savic@exeter.ac.uk

$10{ }^{3}$ Professor, College of Engineering, Mathematics and Physical Sciences, University of 11 Exeter, Exeter, EX4 4QF, UK. E-mail: z.kapelan@exeter.ac.uk

$12{ }^{4}$ Assistant Professor, Dipartimento di Ingegneria Civile ed Architettura, University of Pavia, 13 Via Ferrata 3, 27100 Pavia, Italy. E-mail: creaco@unipv.it

$14{ }^{5}$ Professor, State Key Laboratory of Urban Water Resource and Environment, School of 15 Municipal and Environmental Engineering, Harbin Institute of Technology, Harbin, 150090, 16 China. E-mail: yyx1957@163.com

17 Corresponding author: Haixing Liu

18 Email:lhx_526@163.com 


\section{Abstract:}

20 Due to the large computational burden associated with the direct assessment of reliability, the

21 indirect indices of reliability have recently received more attention in the framework of water

22 distribution system design. Two new energy related indices for reliability evaluation (i.e.,

23 available power index and pipe hydraulic resilience index) are developed in this paper. The

24 performance of these new indices is evaluated and compared with that of four existing indices

25 (other three energy related indices, i.e., resilience index, network resilience index, modified

26 resilience index, and the entropy-based method, i.e., diameter-sensitive flow entropy)

27 according to the following two-step methodology. In the first step, the application of the

28 multi-objective optimization makes it possible to determine optimal network configurations

29 that trade-off the installation cost (to be minimized) against the generic indirect reliability

30 index (to be maximized). In the second step, the performance of the optimal solutions in

31 terms of explicit reliability assessment is examined under conditions in which the original

32 network is perturbed by applying demand variations and random pipe failures to account for

33 future operating uncertainties. The Hanoi and the Fossolo benchmark networks are used as

34 case studies. The results obtained show that energy based indices yield an overall superior

35 estimate of reliability in comparison with the diameter-sensitive flow entropy. Furthermore,

36 the new indices show some advantages in the evaluations performed under demand and pipe

37 failure uncertainties. 
38 Keywords: water distribution system, reliability surrogate measure, available power index, pipe hydraulic resilience index, flow entropy.

\section{Introduction}

41 Traditionally, the optimal design of water distribution systems (WDSs) in the single objective

42 optimisation framework is to obtain the least cost solution in which optional pipe diameters

43 are usually the decision variables and the water sources and layout are known a priori [Savic

44 and Walters, 1997]. In the drive to improve cost effectiveness and ensure water security, the

45 WDS design should incorporate many more aspects [Walski, 2001]. A multiobjective optimisation model generally takes cost as a common objective and combines one or more

47 benefit objectives, such as reliability, robustness, water quality, into a trade-off relationship

48 [Farmani et al., 2005; Kapelan et al., 2005; Creaco and Franchini, 2012; Ostfeld, 2012;

49 Marchi et al., 2014]. Reliability can be classified into three categories: mechanical reliability, hydraulic reliability, and water quality reliability [Ostfeld, 2004; Atkinson et al., 2014; Shafiqul Islam et al., 2014; Gheisi and Naser, 2015]. Reliability is defined as the ability of a WDS to provide an adequate level of service to customers under both normal and abnormal operating conditions within a prescribed time interval [Xu and Goulter, 1999]. Although Jung et al. [2014] argued that robustness definition covers additional information that assesses not only the probability of pressure meeting requirement but also the variation in pressure, reliability undoubtedly remains the most widely applied method for the assessment of WDS performance and is substantially investigated by the scientific community [Dandy and 
58 Engelhardt, 2006; Martinez, 2010; Ostfeld, 2012; Piratla and Ariaratnam, 2012; Torii and

59 Lopez, 2012; Shafiqul Islam et al., 2014].

60 Disruptions in supply could potentially result in a financial loss to a WDS operator. The

61

62

63

64

65

66

67

68

69

70

71

72 concern of how to improve reliability of supply during the design phase is vital. Fujiwara and Tung [1991] stated that reliability could be improved by increasing pipe size. Additionally, tanks play an important role in reliability improvement studies as they help balance water supply and demand in the system, as well as supply water during an emergency situation [Farmani et al., 2005; van Zyl et al., 2008]. Walski [1993] highlighted the importance of adequate isolation valve systems for providing reliability for WDS. Other methods, e.g., adaptive pump operation, multiple sources, are also important for reliability assessment [Zhuang et al., 2013].

Reliability is usually assessed using a probabilistic approach, such as Monte Carlo Simulation, First Order Reliability Method and/or the Cut-set method [Park et al., 1998]. These methodologies are computationally expensive because the assessment involves sampling methods or a traversing process across all combinations of pipe failure. Thus they are difficult to implement within the multiobjective optimisation design methods for a real WDS [Bao and Mays, 1990; Maier et al., 2001; Kapelan et al., 2005; Yannopoulos and Spiliotis, 2013]. As WDS is a complex system and changes over time, the network design that is assessed to be reliable at the design stage is likely to become unreliable in meeting the performance requirements in the future. Another related concept - resilience - can be used to assess reliability indirectly in both conventional [Farmani et al., 2005] and adaptive WDS 
design [Basupi and Kapelan 2013]. Resilience is defined as the ability of system to 'bounce

80

81

82

83

84

85

86

87

88

89

90

91

92

93

94

95

96

97

98

99

back' following some failure and hence can be estimated as the capacity of a system to absorb disturbance while the system is undergoing changes so as to still retain essentially the same function, structure, identity and feedbacks [Folke, 2006]. Todini [2000] proposed the surplus power at nodes to characterise the resilience of looped water networks. Many improvements and applications associated with Todini's resilience index appeared thereafter [Prasad and Park, 2004; Farmani et al., 2005; Jayaram and Srinivasan, 2008; Basupi et al., 2013]. Creaco et al. [2014] showed that a good indication of the network reliability under such critical operation scenarios as those related to pipe isolation and hydrant activation can be obtained when the multi-objective optimization is performed considering three objective functions: 1) cost to be minimized; 2) Todini's resilience to be maximized; 3) loop diameter uniformity to be maximized. Despite the advantages of the methodology proposed by Creaco et al. [2014], the use of three objectives may be too cumbersome in the optimization context for a large network problem.

Other studies have developed indices to assess reliability of water supply. Flow entropy characterising uniformity of pipe flows can be employed to assess reliability of a WDS [Awumah et al., 1990; Tanyimboh and Templeman, 2000; Liu et al., 2014]. Park et al. [1998] introduced an indicator of hydraulic power capacity, which combines flow and pressure in a single dimension for measuring capacity (or reliability) of a WDS. It is, therefore, of interest to researchers and practitioners to determine which reliability assessment method is the most appropriate measure for optimal design studies. 
100 Raad et al. [2010] conducted a comparative analysis of four reliability surrogate measures 101 based on the assumptions of demand and pipe failure uncertainties. They only considered one 102 assessment indicator, i.e., the average demand satisfaction as a result of the demand 103 variability and pipe failure consequence analyses. Baños et al. [2011] compared three existing 104 reliability indices by using the indicators of average percentage of unfeasible scenarios and 105 average minimum over-demand in the scheme of demand uncertainty. Most recently, 106 Atkinson et al. [2014] investigated the correlations amongst resilience index, entropy and 107 minimum surplus head, and examined the hydraulic performance of the solutions for the 108 Anytown benchmark network.

109 Despite the many existing contributions to the field, the assessment of the best reliability 110 surrogate measure(s) is still an open issue. In this paper, two new measures are proposed and 111 their performance compared to four existing measures in the context of the multiobjective 112 design of water distribution systems. In particular, a novel a posteriori performance 113 assessment is used to analyse and compare the surrogate measures in two benchmark 114 networks.

115 The rest of the paper is organized as follows. Firstly, the surrogate measures (including two 116 newly developed) are presented. Secondly, the optimization process and an a posteriori 117 performance assessment method are described. Lastly, the applications are demonstrated and 118 the conclusions drawn. Note that several abbreviations are used in this paper. A list of these 119 abbreviations can be found in Table 1. 
120 Reliability surrogate measures

121 In this study, three existing reliability surrogate measures from literature are analysed,

122 including the resilience index (RI) [Todini, 2000], network resilience index (NRI) [Prasad

123 and Park, 2004] and modified resilience index (MRI) [Jayaram and Srinivasan, 2008]. The

124 above three measures are all derived from the power analysis of water systems. The 125 diameter-sensitive flow entropy (DSFE), newly introduced reliability index based on an

126 extension of the flow entropy formulation [Liu et al., 2014], is also considered. Furthermore,

127 the analysis is also extended to two new measures, the available power index (API) and the

128 pipe hydraulic resilience index (PHRI), which belong to the classes of the power and pipe

129 hydraulic gradient measures, respectively.

130 The following subsections report the formulations of the two new measures (API and PHRI).

131 The other four surrogate measures (RI, NRI, MRI and DSFE) are reviewed in the

132 supplementary material.

\section{Available power index}

134 A WDS is a dynamic, constantly changing and balanced system, which obeys the energy 135 conservation law. The input power (energy per unit time) is derived from sources, pumps and 136 tanks located in high elevations, while the output power could be divided into the available 137 and unavailable power. The available power represents the output power at demand nodes;

138 the unavailable power includes pipe friction losses, minor losses from pumps and bends, and 139 leakage. The output power function $\left(P_{\text {out }}\right)$ is given by 
141 where $P_{a v a}$ is the available portion of output power; $P_{\text {unava }}$ is the unavailable portion.

142 A WDS with higher reliability has the additional capacity to resist the perturbation caused by 143 external uncertainty sources. An obvious way to improve reliability would be to provide a

144 high level of available power while reducing the unavailable power as much as possible. In 145 this paper, a new method, the available power index, is introduced to measure reliability, 146 which is defined as a ratio of the available power $\left(P_{\text {ava }}\right)$ to the total output power $\left(P_{\text {out }}\right)$. The 147 available power index is formulated as,

$$
A P I=\frac{P_{\text {ava }}}{P_{\text {out }}}
$$

149 The available power is delivered to users, which is calculated in terms of flow $\left(Q_{i}\right)$ and head $150\left(H_{a v a, i}\right)$ at each demand node, shown as follows,

$$
P_{a v a}=\gamma \sum_{i=1}^{\text {nnode }} Q_{i} H_{a v a, i}
$$

152 The input power $\left(P_{i n}\right)$ includes the power introduced into the network by pumps $\left(P_{k}\right)$ and 153 provided by reservoirs and tanks.

$$
P_{i n}=\gamma \sum_{j=1}^{\text {nreservoir }} Q_{j} H_{j}+\sum_{k=1}^{n p u m p} P_{k}+\gamma \sum_{l=1}^{\text {ntank }} Q_{l} H_{l}
$$

155 where $Q_{l}$ is the tank flow into or out of the network and $H_{l}$ is the free surface elevation at $156 \operatorname{tank} l$; ntank is the number of tanks. The flow of tank is positive when the water comes 
out of the tank, and vice versa. Due to the input power equal to the output power $\left(P_{i n}=P_{\text {out }}\right)$,

158 Equation 2 could be substituted with Equation 3 and 4. The available power index is written 159 as,

160

$$
A P I=\frac{\sum_{i=1}^{\text {nnode }} Q_{i} H_{\text {ava }, i}}{\sum_{j=1}^{\text {nreservoir }} Q_{j} H_{j}+\sum_{k=1}^{n \text { pump }} \frac{P_{k}}{\gamma}+\sum_{l=1}^{\text {ntank }} Q_{l} H_{l}}
$$

161 A tank can be viewed as a feeding or demanding node. The tank in Equation 5 is put in the supply term, since the tank power avoids the denominator becoming zero when the pump does not supply water to the pressurized system. The available power index is the limiting

164 form of Todini's resilience index, i.e., RI's required head equal to zero. However, they have entirely different physical meanings. API is based on the analysis of the energy conservation principle and is expressed as the available power in a WDS, while the RI concept emphasizes

167 the surplus power in the system. In addition, due to requiring one parameter less (i.e., the 168 required head at demand nodes), the available power index is more straightforward and readily computed in comparison with the resilience index. Furthermore, the available power index explicitly expresses the significance of tanks. This is essential, since tanks not only

171 balance the flow difference between on-peak and off-peak periods, but also store energy

172 during normal operation and emergency situations (e.g., fire fighting, pipe breaks). Hence

173 tanks play a key role of energy transfer within a WDS are necessary in the analysis of the available power. 
175

176 The aforementioned methods associated with resilience of WDSs belong to a same class in

177 which power at nodes is taken into account. A new method in terms of the hydraulic gradient

178 along pipes is developed and presented here. Hydraulic gradient is related to the head

179 difference at both ends of a pipe and the pipe length. When water flows through a pipe, the

180 hydraulic grade line (HGL) will decline due to friction loss, as shown in Figure 1. If upstream

181 pipes dissipate less energy, the downstream pipes will obtain more head. The magnitude of

182 available head could reflect the capacity of a WDS to handle the perturbation caused by

183 uncertainty. However, available heads are insufficient to quantify reliability on their own, as

184 one metre head loss in a pipe of 100-metre length differs from that of a one-kilometre long

185 pipe. Hence, a new measure for WDS resilience which combines pressure head and pipe

186 length is developed.

187 The product of pressure head and pipe length is represented by an area, as shown in Figure 1.

188 The blue triangle area $\left(A_{i}\right)$ is characterized by the head loss and the projection length of the 189 pipe, while the red triangle area $\left(S_{i}\right)$ is determined by the available head and the projection 190 length of the pipe. Pipe hydraulic resilience index is defined as

191

$$
\text { PHRI }=\frac{\sum_{i=1}^{n} S_{i}}{\sum_{i=1}^{n}\left(A_{i}+S_{i}\right)}
$$


where $n$ is the number of pipes in the WDS. It is worth noting that the areas are summed up prior to being divided in order to avoid simultaneous reduction of pipe length. The functions

194 of area $S_{i}, S_{i}+A_{i}$ and the projection length of pipe $i\left(L_{p r o, i}\right)$ are expressed as follows,

$$
\begin{aligned}
& S_{i}=\frac{1}{2}\left(H_{d s, i}-H_{r e q}\right) L_{p r o, i} \\
& S_{i}+A_{i}=\frac{1}{2}\left(H_{u s, i}-H_{r e q}\right) L_{p r o, i} \\
& L_{p r o, i}=\sqrt{L_{i}^{2}-\left(Z_{u s, i}-Z_{d s, i}\right)^{2}}
\end{aligned}
$$

198 where $H_{d s, i}$ and $H_{u s, i}$ are the heads at downstream and upstream nodes, respectively, for 199 pipe $i ; H_{r e q}$ is the required head of the system; $L_{i}$ and $L_{p r o, i}$ are the length and the 200 projection length, respectively, for pipe $i . Z_{d s, i}$ and $Z_{u s, i}$ are the elevations at downstream and upstream nodes for pipe $i$.

\section{Assessment and comparison methodology}

203 A comprehensive framework is established here to evaluate and compare the surrogate 204 reliability measures. The assessment procedure is based on a set of solutions derived from the 205 optimal design of WDSs associated with each reliability measure. The design samples (i.e., 206 the optimal solutions of network design) are tested in scenarios perturbed by demand spatial 207 fluctuation and random pipe failure uncertainties. The evaluation indicators are proposed to assess the performances of the design samples, and then determine the performance of reliability surrogate measures. 
210 The sample of WDS designs is obtained by applying a multiobjective optimisation process.

211 Each of reliability surrogate measures introduced above is respectively used as a conflicting

212 objective against the WDS design cost. The optional diameters of new pipes are the only

213 decision variables. A well-known multiobjective optimisation algorithm, the elitist

214 Non-dominated Sorting Genetic Algorithm II (NSGA-II), is used to solve this problem [Deb

215 et al., 2002]. A modified version of EPANET2 [Rossman, 2000] is used as the hydraulic

216 engine. In order to compare the performance of the six different surrogate measures, the

217 optimal solutions for each pair of surrogate reliability and cost (i.e. six different formulations)

218 are respectively derived by the optimisation process. The same optimization process is

219 conducted ten times for each of the six formulations to avoid the influence of the randomness

220 of generating initial solutions. Ten sets of non-dominated solutions are aggregated to yield an

221 optimal solution set by using Pareto dominance concept.

\section{WDS performance in perturbed scenarios}

223 The uncertainty of WDSs stems from a variety of aspects, while in this paper the two

224 common uncertainties of demand spatial fluctuation and pipe random failure are considered.

225 Probabilistic methods are typically used for handling uncertainty analysis in WDSs [Kapelan

226 et al., 2005; Filion et al., 2007; Sumer and Lansey, 2009; Fu and Kapelan, 2011]. Moreover, a

227 marginal quantity method could be used to quantify the ability of systems to withstand

228 uncertain incidents. The marginal quantity could be represented by a number or a polyhedron

229 [Housh et al., 2011; Sun et al., 2011]. In order to establish the accessible and versatile

230 evaluation indicators for reliability, the probabilistic method is still applied herein. 
231 The demand uncertainty is often characterised by an appropriate probability density function 232 (PDF) through a considerable amount of sampling [Fu and Kapelan, 2011]. Many researchers

233 conducted the analysis for demand fluctuation in the assumption of uncertainty following 234 various PDFs including normal, log normal, uniform, $\beta$, Pearson type III, to name a few [Bao 235 and Mays, 1990; Giustolisi et al., 2009; Kang et al., 2009]. The normal distribution is used 236 most frequently out of those mentioned above, and thus is adopted in this paper. The mean of 237 normal distribution is equal to the design demand. The coefficient of variation is assumed to 238 be 0.5 , which implies a wide range of demand variation, e.g., when the change of usage type 239 occurs due to area development. The Latin Hypercube Sampling (LHS) is used to sample 240 varying demand in terms of the PDF for each node. The Latin Hypercube Sampling (LHS)

241 technique is selected here to reduce the computational effort substantially in comparison with 242 the Monte Carlo sampling procedure [Kang et al., 2009]. LHS is implemented based on 1,000 243 samples in this study.

244 The second part of the assessment framework for reliability surrogate measures is established 245 using perturbation related to pipe failure. Under the assumption that a failed pipe can be 246 specified and isolated, the procedure involves the assessment of the system performance. To 247 this end a single pipe isolation is considered, since the probability of simultaneous occurrence 248 of two pipes failure is extremely low, and the procedure then traverses each pipe one by one 249 across the network. Raad et al. [2010] conducted the comparison of reliability measures 250 associated with a single pipe failure under the assumption of the equal probability of failure 251 for all pipes. However, the failure probability of pipes with large diameters is much lower 
252 than in the case of small-diameter ones, since former have thicker walls and are usually better 253 maintained (due to higher importance in the system). The new method combines the 254 probability of pipe failure with the reliability evaluation indicators. Some common 255 assumptions with respect to pipe failure are stated as follows:

1) N-type isolation valves: There are isolation valves at both ends of every pipe, and thus the isolation segment is just limited to a pipe;

2) Simulation of a failed pipe: A pipe outage is simulated by closing the corresponding link in the model; and

3) Nodal demand: Water is distributed along pipes. However, it is common modelling practice to aggregate demand at nodes. When a given pipe is closed, the reduced quantity supplied by that pipe is ignored.

The testing for demand fluctuation and pipe failure intervention are both based on the same set of design solutions for each reliability surrogate measure, but they are independent and executed separately.

\section{Reliability evaluation indicators}

The best performance associated with reliability is achieved when the solution sample is able to sustain in a normal operation/level of service under various kinds of perturbation or have the least impact on the performance under the failed scenarios. A failed scenario refers to the case when there is at least one failed node in the system, i.e., such that it cannot achieve the prescribed level of service (supplied water cannot meet the demand). Farmani et al. [2005] 
272 indicated that a reliability measure should reflect the way in which water users are affected,

273 i.e., the number of users with restricted or no service and the degree (magnitude) of the effect

274 on the service level. Improved assessment indicators are introduced in this study, consisting 275 of:

- The sum of the number of scenarios with at least one failed node;

- The spatial extent of failed nodes in the network, which is represented by a failed node count under a particular failed scenario;

- The failure magnitude associated with the level of service at nodes.

In order to evaluate these indicators, pressure driven demand (PDD) needs to be integrated into the WDS hydraulic solver. The modified version of EPANET2 [Morley and Tricarico, 2008] is implemented in this study. Three global indicators of system performance are proposed as follows: PFS function returns the percentage of the total evaluated scenarios in which the network fails to achieve the prescribed demand target for at least one node. total number of failed nodes where the demand at nodes does not meet the required level of service. For all failed scenarios of a given optimal solution, the average of FNC $\left(F N C_{\text {mean }}\right)$, the median of FNC $\left(F N C_{\text {median }}\right)$, the $25^{\text {th }}$ percentile of FNC $\left(F N C_{25 t h}\right)$ and the $75^{\text {th }}$ percentile of FNC $\left(F N C_{75 t h}\right)$ could be calculated. 

of the inadequate amount of water (i.e. the difference between the demand and the actual supplied water at a node) to the sum of the water required. Similarly, some statistics with respect to the FFD indicator are proposed: the average of FFD $\left(F F D_{\text {mean }}\right)$, the median of FFD $\left(F F D_{\text {median }}\right)$, the $25^{\text {th }}$ percentile of FFD $\left(F F D_{25 t h}\right)$ and the $75^{\text {th }}$ percentile of FFD $\left(F F D_{75 t h}\right)$.

298 The greater the values of PFS, FNC and FFD, the greater the impact of perturbation on the 299 network is, and thus the worse the performance of reliability surrogate measure. A method for 300 evaluating overall performance of all solutions of a surrogate measure is proposed, which is 301 defined as an integral of an indicator value at a fixed cost step. In detail, the integral method 302 is implemented by the sum of the indicator values for each solution multiplied by the cost 303 step between two consecutive solutions. The smaller the integral value, the better the overall 304 performance of the reliability surrogate measure is. The integral method is mathematically 305 expressed as follows:

$$
O P=\int_{\omega} f\left(P_{\text {indicator }}\right) \mathrm{dP}(\omega)
$$

307 where $O P$ is the overall performance of an indicator at a range of costs; $\mathbb{P}(\omega)$ is the 308 vector space of costs; $\omega$ is the cost variable; $f\left(P_{\text {indicator }}\right)$ represents the statistical method 309 of an indicator; $P_{\text {indicator }}$ represents the value of a reliability evaluation indicator 
310 Case studies

\section{Benchmark networks}

312 We use two different case studies, the Hanoi and Fossolo benchmark networks, to

313 demonstrate and test our methodology. The comparative analysis of reliability surrogate

314 measures in the first case study is based on a modified benchmark network [Fujiwara and

315 Khang, 1990]. The original Hanoi network is supplemented by a tank with an initial head of

$31680 \mathrm{~m}$ which is connected to the network by a 100m-long pipe, as shown in Figure 2(a). The

317 network is flat as the elevation of all nodes (apart from the elevated tank) are the same. The

318 head at all nodes of the network is required to be larger than or equal to $30 \mathrm{~m}$. The search

319 space for the Hanoi network design problem is equal to $2.87 \times 10^{26}$.

320 The second case study is a benchmark network adopted from Wang et al. [2014]. The Fossolo

321 network, which includes 58 pipes, 36 demand nodes, and one reservoir with a fixed head of

$322121 \mathrm{~m}$, is a much more complex design problem to solve as its search space is equal to $7.25 \mathrm{x}$

$32310^{77}$. The Fossolo network shows a variation of node elevation, ranging from $61.24 \mathrm{~m}$ to

$32467.90 \mathrm{~m}$. The minimum pressure head of all the demand nodes is to be maintained at or above

$32540 \mathrm{~m}$, and a maximum pressure head is specified for each node, respectively. In addition, the

326 flow velocity in each pipe should be no greater than $1 \mathrm{~m} / \mathrm{s}$. Figure 2(b) depicts the layout of

327 the network. Both networks use the same cost objective, given as:

328

$$
\text { Cost }=\sum_{i=1}^{N} U_{c}\left(D_{i}\right) L_{i}
$$


where $U_{c}$ is the unit cost of pipe $i$ which is a function of diameter $D_{i} ; L_{i}$ is the length of pipe $i ; N$ is the total number of pipes.

\section{Sample of Solutions}

332 Optimizations are carried out considering, as objective functions, the network cost and each

333 of the six reliability measures described in the methodology section. In particular, for each

334 pair of objective functions (i.e., one of the reliability surrogate measures and cost), a set of 10

335 optimization runs is carried out in order to explore the influence of randomly generated initial

336 populations. For the Hanoi network, a preliminary sensitivity analysis led to the choice of a

337 population of 200 individuals and of a total of 500 generations for the optimization sets with

338 RI, NRI, MRI, API and PHRI. Additional analyses determined that optimisation runs with

339 these NSGA-II parameters and using DSFE were not converging. Therefore, a population of

340200 individuals and a total of 5,000 generations were allowed for the optimization set with

341 DSFE. For the Fossolo network, the parameters used for the optimization are the population

342 of 200 individuals and 5,000 generations per run, while 10,000 generations were allowed for

343 the runs using the DSFE measure.

344 The optimal solutions are generated using the Pareto dominance approach by aggregating

345 best solutions from all ten optimization runs. This was done to ensure that as optimal as

346 possible Pareto fronts are obtained and compared. The solution samples for Hanoi are

347 selected from the overlapping area of costs, as shown in Figure 3, in order to implement the

348 integral method and provide a fair comparison. The values of RI, NRI, API and PHRI range

349 from 0 to 1 , while MRI and DSFE only have a lower bound (i.e., 0). The Pareto front of 
350 DSFE is less smooth when compared to other fronts, as a consequence of the more 351 complicated search for solutions in this case. The Pareto optimal solutions for Fossolo are

352 shown in Figure 4(a), and the shared cost solutions in the range from 0.03 to 0.7 million

353 dollars are selected as samples to be evaluated. The Figure 4(b) represents the enlarged area

354 from Figure 4(a).

355 The comparative analysis of the surrogate measures is fair on the premise of the same

356 convergence of the optimization process. For both networks used in this paper, the near

357 optimal solutions were validated in comparison with the results obtained by Wang et al [2014]

358 where they used massive computational resources in order to achieve the best-know

359 approximation of the true Pareto front.

\section{Comparison of results: Case \#1}

\section{$361 \quad$ Perturbation of demand}

362 The samples of the solutions obtained in the previous section are evaluated first using the

363 indicators of PFS, FNC and FFD under demand perturbation. In Figure 5, $F N C_{\text {mean }}$, $364 F N C_{\text {median }}, F N C_{25 t h}$ and $F N C_{75 t h}$ are calculated for each solution. For all surrogate 365 measures, the extent of FNC variation tends to be small and $F N C_{75 t h}$ decreases with the

366 increase in cost in the testing of demand perturbation. The reason is that the system with 367 higher cost has consistently greater redundancy to cope with demand changes for all 368 surrogate measures. Obviously, the values of FNC (i.e., the number of the failed nodes) of RI, 369 NRI, MRI and API decline steeply for lower costs. As a result, when RI, NRI, MRI and API 370 measures are used to investigate reliability, increasing the capital investment at the lower 
371 costs will have a significant effect on mitigating the affected (failure) area under demand 372 perturbation. It is interesting to observe that $F N C_{\text {mean }}$ of DSFE remains at an almost 373 constant value of about nine failure nodes across all the costs in Figure 5(f). The increased 374 cost is not necessarily beneficial for reducing the impact of demand perturbation on the 375 failure extent captured by the DSFE measure. The vast majority of $F N C_{\text {median }}$ values of 376 DSFE are greater than $F N C_{\text {mean }}$. More values are clustered toward the upper end of the range 377 or few extreme values at the lower end are causing this skewness. For the network, a few 378 cases of demand fluctuations have a less significant impact on the spatial extent of the 379 deficient nodes.

380 The indicator of FFD is a widely used measure, and in this assessment framework, FFD 381 represents the inadequate degree of water delivered at failed nodes. FFD statistics for all 382 solutions are shown in Figure 6. The overall trend is that FFD values decline with the cost 383 increase except for DSFE. Unlike the FNC, the trends of $F F D_{25 t h}$ and $F F D_{75 t h}$ are 384 relatively consistent. The FFD values (i.e., the fraction of failure degree) of RI, NRI, MRI 385 and API experience no significant change for the lower cost values, while the FNC values of 386 these solutions decrease dramatically. This implies that, among these solutions, the spatial 387 extent of failed nodes has not expanded, but the failure degree of these failed nodes becomes 388 more severe. The failure degree of PHRI at failed nodes declines throughout all the costs. 389 The FFD statistics of DSFE are approximately invariable.

390 Reliability surrogate measures are analysed using the integral method (i.e., Equation 10) 391 based on two kinds of scale: 1) one-segment cost step for which the integral procedure is 
392 calculated once throughout the cost values; and 2) five-segment cost step for which the 393 integral calculation will be conducted at each fifth of the cost interval. The computation at 394 two scales is based on Equation 10, and the difference is that the one fifth range of cost is 395 respectively calculated in the five-segment integral. The integral results under demand 396 uncertainty perturbation are shown in Figure 7. The one-segment integral is plotted as light 397 grey columns, and that of five-segment results are represented by a line and symbol plot. 398 Figure 7(a) shows that the PFS values of PHRI are the lowest in the low cost region, but they 399 grow with the increase in cost. The figure also shows that the PHRI solutions with the smaller 400 diameter redundancy have the smallest failure scenario count and the strongest resistance 401 capacity with respect to the perturbation caused by demand variation. Overall, the PHRI 402 solutions have the smallest failure scenario count in terms of the 1-segment integral. DSFE 403 performs poorly compared with other surrogate measures throughout the entire cost spectrum. 404 RI, NRI, MRI and API have the similar PFS values for the five-segment cost steps. In the 405 comparison for 1-segment cost step, the NRI is slightly better than RI, MRI and API (see 406 Figure 7(d).

407 It is worth noting that the FNC statistics are only considered based on the failed scenarios. 408 For PHRI solutions, the probability of occurrence of failed scenarios associated with demand 409 perturbation is lower (see Figure 7d), but there will be a greater extent of impact on system 410 when a failure occurs (see Figure 7e).

411 The values of $F N C_{\text {mean }}$ are used to calculate the integral of FNC, whose results are shown as 412 Figure 7(b) and (e). The smallest value of 1-segment integral of FNC is derived from RI. 
413 However, the RI measure shows the lowest integral values at the higher cost steps for

414 5-segement integral of FNC in comparison with NRI, MRI and API. NRI has the greatest

415 mean value of failure nodes among the power-related family of surrogate measures. The

416 measures of PHRI and DSFE show the larger extent of impact under demand perturbation.

417 The mean FFD related to demand uncertainty is used to calculate the integral of FFD, as

418 shown in Figure 7(c) and (f). PHRI has the smallest integral value of FFD for 1-segment. The

419 analysis of the 5-segment integral shows that the PHRI plays a dominant role for the lower

420 cost region in comparison to other surrogate measures. NRI is the second best for failure

421 degree at failed nodes. Again, DSFE does not perform well and has an increasing difference

422 from other indices towards the high cost.

423 Perturbation of pipe failure

424 Pipe failure uncertainty is considered in this paper by using the concept of network 425 availability [Cullinane et al., 1992], which is defined as the proportion of the time that the 426 system will satisfactorily fulfil its function. Only the FFD indicator involves the network 427 availability, since PFS and FNC indicate the actual number of failed scenarios and nodes, 428 respectively. All failure scenarios considered are based on the fully connected network 429 configurations.

430 The failure node count values for each solution are shown in Figure 8. There are similar, 431 relatively flat patterns of variation for FNC statistics amongst RI, NRI, MRI, API and PHRI.

432 The figure also shows that most of solutions have a relatively small number of failed nodes 433 and some critical nodes are significantly influenced by pipe failure. The $F N C_{\text {median }}$ values 
434 are lower than $F N C_{\text {mean }}$ (Figure 8a-8f). This skewness is inverse in comparison with the

435 results of FNC in demand perturbation scenarios (Figure 5). It demonstrates that several

436 critical pipe failures could result in a great number of nodes affected (failed). It can be

437 observed that $F N C_{75 t h}$ of PHRI declines slightly as the cost increases in Figure 8(e).

438 In Figure 9, the failure degree at failed nodes declines with the increase in cost for all 439 reliability surrogate measures. However, the failed node count does not decrease in the pipe

440 failure testing in Figure 8. The downward trend of FFD can be attributed to the cost increase,

441 either larger diameter pipe leading to a lower probability of pipe failure or conveying more

442 water to failed nodes through other pathways. Although it is difficult to identify which is the

443 dominant reason, this trend reflects better the practical situation. The declining trends of $444 F_{25 D^{2 h}}$ and $F F D_{75 t h}$ are consistent throughout the cost range for RI, NRI, MRI, API,

445 PHRI (see Figure 9a-9e). The redundancy of pipes increases consistently with increases in

446 cost. However, with reference to DSFE, some of pipes taken out of service could lead to the 447 severely decreased system performance (see Figure 9f).

448 The integral of PFS is shown in Figure 10(a) and (d). The trends of all surrogate measures 449 show a decline with the increase in cost, which illustrates that by increasing capital cost one 450 can reduce the impact of pipe failure on WDSs. The comparison of surrogate measures 451 demonstrates that API has the lowest value of 1-segment integral and the lowest failure 452 scenario count under pipe failure perturbation. RI has the second lower value for the PFS 453 1-segment integral. There is a similar situation with DSFE for the case of demand uncertainty. 
454 The failure scenario count of DSFE shows the similar results with other measures at the 455 lowest cost step, but the DSFE has great differences at other cost steps.

$456 F N C_{\text {mean }}$ is used as a representative value to calculate the integral of FNC, as shown in

457 Figure 10(b) and (e). PHRI has the best performance with respect to failure node count for

458 1-segment integral and outperforms other indices almost at all cost steps for 5-segment

459 integral. In the power family of surrogate measures, MRI obtains a better performance than

460 other power-related indices. Once again, DSFE has the greatest number of failed node count.

461 The integral of $F F D_{\text {mean }}$ is shown in Figure 10 (c) and (f). The integral values of NRI and

462 DSFE for 1-segment are relatively small. However, they are not ideal options since DSFE 463 values vary significantly and the NRI measure outperforms other indices only at the highest

464 cost step in the 5-segment integral. By the comparison of Figure 7 and 10, DSFE seems to 465 perform better in resisting the perturbation of pipe failure than the disturbance of demand 466 changes due to taking into account the impact of diameters on reliability.

\section{Comparison of results: Case \#2}

$468 \quad$ Perturbation of demand

469 The Pareto optimal solutions for the Fossolo network are derived via the previously described

470 optimization process. The assessment associated with demand and pipe failure perturbations

471 is performed post optimization based on the obtained optimal solutions. The assessment

472 results are represented by a set of indicator statistics. The indicator based on the failure node

473 count (FNC) is shown in Figure $\mathrm{S} 1$ in the supplementary material. For RI, NRI, MRI, API

474 and PHRI, the FNC values declines sharply at the lower cost, while the changes of the FNC 
475 indicator become relatively flat at the high cost. Meanwhile, the $25^{\text {th }}$ percentile and $75^{\text {th }}$

476 percentile and median merge together with the increase in cost. This indicates that the

477 solutions at the high cost end have only one or no failed scenarios in 1,000 demand

478 fluctuation evaluations, which can also been seen in Figure 11(a). It should be emphasized

479 that the indicators can achieve zero at the highest cost when using RI, NRI, MRI and API

480 surrogate measures, which shows these networks exhibit high reliability with respect to

481 demand fluctuations.

482 The DSFE surrogate measure has the worst performance with respect to the FNC indicator. In

483 the case of increasing cost, the networks derived using DSFE still have a large number of

484 failed nodes. However, the range of variation of FNC is small for DSFE. For the NRI

485 measure, the networks with the higher cost exhibit a wide range of changes of failed nodes. It

486 is worth noting that the Latin Hypercube sampling method uses the same random seed for all

487 surrogate measures, but the NRI solutions at higher costs are not as good as other surrogate

488 measures with respect to the demand perturbation. In other words, the solutions obtained by

489 using NRI show a larger spatial extent of failures under the demand perturbation condition.

490 The indicator of fraction of failure degree (FFD) is calculated and the results of its statistics

491 are shown in Figure S2 in the supplementary material. All the surrogate measures except

492 DSFE exhibit a reduction in failure degree with the increase in cost. Furthermore, at the

493 lower costs, the FFD decreases sharply and then became relatively flat at the higher costs. RI,

494 MRI, API and PHRI have very narrow ranges of variation for the demand perturbation. In

495 contrast, the NRI has a wider range of variation, which demonstrates that although network 
configurations derived from NRI can resist a proportion of the demand perturbation very well,

497 they are less effective for other perturbations. This is likely due to NRI being based on a

498 uniformity coefficient for pipe diameters, which means that diameters in the network tend to

499 increase evenly from the source to the extremities. If the increased demands appear near the

500 source, the network can easily handle this perturbation. However, if the increased demands

501 are applied at the nodes far away from the source, it is most likely to result in a failed

502 scenarios. Different from source-bias diameter layout of the NRI, it is common engineering

503 practice that larger diameters are associated with trunk mains, and the relatively small

504 diameters are deployed as offshoots from the trunks.

505 The integral method is used to compare the surrogate measures. The integral results of the

506 Fossolo network is given in Figure 11. The DSFE surrogate has the largest values for all three

507 indicators. It should, however, be noted that DSFE solutions with smaller costs (basically in

508 the first cost segment) are competitive with other surrogate measures, but when the cost

509 increases, it becomes inferior.

510 NRI is the second worse measure when demand perturbations are applied to the Fossolo

511 network. This is probably because NRI tends to favour diameter redundancy (i.e., larger

512 diameters) near the source of the network. However, the demand perturbations are applied

513 uniformly across the network. As a results, NRI's bias for diameter redundancy fails to

514 perform well for this kind of global demand perturbations. 
515 The surrogate measures of RI, MRI and API show similar behaviour to each other under

516 demand perturbations. The newly developed PHRI outperforms other measures with respect

517 to other indicators in the number of failed scenarios. It could be seen in Figure 11(d) that the

518 tiny advantage is stemmed from the second cost interval in the five-segment integral. But

519 PHRI becomes slightly inferior for other two indicators.

\section{Perturbation of pipe failure}

521 Failure node count is calculated next with respect to the pipe failure perturbation, as shown in

522 Figure S3 in the supplementary material. The RI, MRI, and API measures show similar

523 behaviour in terms of FNC values such that with the increase in cost, the number of failed

524 nodes decreases generally. Here, the variation of FNC is different from its behaviour shown

525 in the demand perturbation graphs. However, the non-monotonic decreasing trend is the same

526 as in the pipe failure perturbation case in the Hanoi network. The pipe faliure perturbation

527 ususlly causes the node failure at the downstream of the incident pipe, and these failed nodes

528 tend to locate at the extremities. Hence, it indicates that the pipe failure often fails the critical

529 nodes in a certain extent.

530 The values of the FNC indicator exhibit significant changes at the lower cost values for PHRI

531 and NRI. And, at most higher costs, the FNC values of PHRI and NRI are eqaul to zero. This

532 indicates that there is no node that fails to meet the requirement in the network, no matter

533 which pipe is taken out of service. On the contrary, DSFE does not show an obvious trend

534 regarding the FNC indicator. 
535 The fraction of failure degree indicator (FFD) is shown in Figure S4 in the supplementary 536 material. All surrogate measures, except DSFE, show a similar trend in which FFD decreases

537 with the increase in cost. Particularly, NRI and PHRI achieve a good performance for the 538 pipe failure perturbation. However, these two surrogate measures are worse than RI, MRI and 539 API in the assessment of demand perturbation in the Fossolo network. This demonstrates that 540 the network designs derived from MRI and PHRI measures are good at coping with local 541 perturbations (i.e., the pipe failure), rather than global perturbations (i.e., demand fluctuation)

542 for this particular network. The range of variation of DSFE is relatively small at the lower 543 costs, but its statistics takes a much wider range at the higher cost end.

544 The integral values are calculated by Equation 10, and the results are shown in Figure 12. 545 With respect to the pipe failure perturbation in the Fossolo network, NRI achieves the best 546 performance for all three indicators, as it obviously has a much lower value than other 547 surrogate measures. PHRI is the second best in the assessment, while RI, MRI and API have 548 similar values in both one segment and five-segment integrals.

549 DSFE has the worst results overall by using the one segment integral analysis, but it can be 550 seen that it facilitates searching for reliable solutions, especially in the lower cost range. 551 Another point which is worth noting is that DSFE's solutions have a better performance in 552 handling the pipe failure perturbation than that for the demand uncertainty perturbation. This 553 can be reflected in both case studies. With a sufficient budget, DSFE tends to deploy multiple, 554 and long, trunk mains in order to achieve uniformly distributed flows based on the flow 555 entropy measure. The pipe flows take a long route to the users, and thus more energy is 
556 dissipated, which leads to a more severe impact once the demand fluctuation and pipe 557 isolation occur. Besides, it may be that the higher cost solutions derived by using DSFE are 558 sub-optimal for this network.

\section{Conclusions}

560 The paper presented two new reliability surrogate measures (i.e., available power index and

561 pipe hydraulic resilience index) and compared these to the four existing measures from the

562 literature (i.e., resilience index, network resilience index, modified resilience index and

563 diameter-sensitive flow entropy). The comparison was done under the conditions of demand

564 and pipe failure uncertainties on the problem of optimal WDS design. The demand

565 uncertainty, characterised using normal distribution, is implemented by a stochastic sampling

566 procedure which simulates different demand spatial fluctuations. The pipe failure uncertainty

567 is described by the existing probability of a pipe failure model. The performance of obtained

568 optimal WDS designs, derived by minimising total cost of interventions and maximising

569 system reliability of supply (achieved by using above reliability surrogate measures, one at a

570 time), is examined by using three indicators: (i) percentage of failure scenarios, which

571 represents the overall performance of a system under uncertain conditions; (ii) failure node

572 count, which indicates the spatial extent of the level of service reduction in failed scenarios;

573 and (iii) the fraction of failure degree, which denotes the magnitude of service deficiency at

574 failed nodes. 
575 The above methodology was applied to the case studies of Hanoi and Fossolo WDSs. The 576 results obtained show that the pipe hydraulic resilience index and available power index

577 proposed performed well under demand and pipe failure perturbations. Opposite of this, the 578 diameter-sensitive flow entropy performed relatively poorly. It is worth noting that the

579 low-cost DSFE solutions performed much better than that at the larger cost. The NRI 580 surrogate measure has a better performance in the pipe failure perturbation than that for the

581 demand fluctuation perturbation. Generally speaking, it seems that the power family of 582 reliability surrogates, including RI, MRI and API, achieved similar results despite small 583 differences in implementation.

584 Further work would be necessary to investigate the reliability surrogate measures in a wide 585 range of large-scale real-world WDSs. Note that not only should surrogate measures assess 586 reliability accurately, but also it is a concern that, in the case of large-scale real-world WDSs, 587 the measure can be implemented in the search of optimal solutions with high computational 588 efficiency.

\section{Acknowledgements}

590 The data of Hanoi and Fossolo networks which were used as the case study can be found on 591 the website of Centre for Water Systems, University of Exeter, UK 592 (http://emps.exeter.ac.uk/engineering/research/cws/resources/benchmarks/). This study is

593 financially supported by the National Natural Science Foundation of China (51178141). 


\section{References}

595 Atkinson, S., R. Farmani, F. Memon and D. Butler (2014). Reliability Indicators for Water

596 Distribution System Design: Comparison, Journal of Water Resources Planning and Management, 597 140(2), 160-168, doi:10.1061/(ASCE)WR.1943-5452.0000304.

598 Awumah, K., I. Goulter and S. Bhatt (1990). Assessment of reliability in water distribution networks 599 using entropy based measures, Stochastic Hydrology and Hydraulics, 4(4), 309-320, 600 doi:10.1007/bf01544084.

601 Baños, R., J. Reca, J. Martínez, C. Gil and A. Márquez (2011). Resilience Indexes for Water 602 Distribution Network Design: A Performance Analysis Under Demand Uncertainty, Water Resources 603 Management, 25(10), 2351-2366, doi: 10.1007/s11269-011-9812-3.

604 Bao, Y. and L. Mays (1990). Model for water distribution system reliability, Journal of Hydraulic 605 Engineering, 116(9), 1119-1137, doi:10.1061/(ASCE)0733-9429(1990)116:9(1119).

606 Basupi, I., Z. Kapelan and D. Butler (2013). Reducing life-cycle carbon footprints in the redesign of 607 water distribution systems, Journal of Water and Climate Change, 4(3), 176-192, 608 doi:10.2166/wcc.2013.004.

609 Creaco, E. and M. Franchini (2012). Fast network multi-objective design algorithm combined with an 610 a posteriori procedure for reliability evaluation under various operational scenarios, Urban Water 611 Journal, 9(6), 385-399, doi: 10.1080/1573062x.2012.690432.

612 Creaco, E., M. Franchini and E. Todini (2014). The combined use of resilience and loop diameter 613 uniformity as a good indirect measure of network reliability, Urban Water Journal, 1-15, doi: 10.1080/1573062X.2014.949799. 
615 Cullinane, M., K. Lansey and L. Mays (1992). Optimization-availability-based design of 616 water-distribution networks, Journal of Hydraulic Engineering, 118(3), 420-441, 617 doi:10.1061/(ASCE)0733-9429(1992)118:3(420).

618 Dandy, G. and M. Engelhardt (2006). Multi-Objective Trade-Offs between Cost and Reliability in the 619 Replacement of Water Mains, Journal of Water Resources Planning and Management, 132(2), 79-88, 620 doi:10.1061/(ASCE)0733-9496(2006)132:2(79).

621 Deb, K., A. Pratap, S. Agarwal and T. Meyarivan (2002). A fast and elitist multiobjective genetic 622 algorithm: NSGA-II, Evolutionary Computation, IEEE Transactions on, 6(2), 182-197, doi: $623 \quad 10.1109 / 4235.996017$.

624 Farmani, R., G. Walters and D. Savic (2005). Trade-off between total cost and reliability for Anytown 625 water distribution network, Journal of Water Resources Planning and Management, 131(3), 161-171, 626 doi:10.1061/(ASCE)0733-9496(2005)131:3(161).

627 Filion, Y., B. Adams and B. Karney (2007). Stochastic Design of Water Distribution Systems with 628 Expected Annual Damages, Journal of Water Resources Planning and Management, 133(3), 244-252, 629 doi:10.1061/(ASCE)0733-9496(2007)133:3(244).

630 Folke, C. (2006). Resilience: The emergence of a perspective for social-ecological systems analyses, 631 Global Environmental Change, 16(3), 253-267, doi: 10.1016/j.gloenvcha.2006.04.002.

$632 \mathrm{Fu}, \mathrm{G}$. and Z. Kapelan (2011). Fuzzy probabilistic design of water distribution networks, Water 633 Resources Research, 47(5), W05538, doi: 10.1029/2010WR009739. 
634 Fujiwara, O. and D. B. Khang (1990). A two-phase decomposition method for optimal design of 635 looped water distribution networks, Water Resources Research, 26(4), 539-549, 636 doi:10.1029/WR026i004p00539.

637 Fujiwara, O. and H. D. Tung (1991). Reliability improvement for water distribution networks through 638 increasing pipe size, Water Resources Research, 27(7), 1395-1402, doi:10.1029/91wr00882.

639 Gheisi, A. and G. Naser (2015). Multistate Reliability of Water-Distribution Systems: Comparison of 640 Surrogate Measures, Journal of Water Resources Planning and Management, 141(10), 04015018, 641 10.1061/(ASCE)WR.1943-5452.0000529.

642 Giustolisi, O., D. Laucelli and A. Colombo (2009). Deterministic versus Stochastic Design of Water

643 Distribution Networks, Journal of Water Resources Planning and Management, 135(2), 117-127, 644 doi:10.1061/(ASCE)0733-9496(2009)135:2(117).

645 Housh, M., A. Ostfeld and U. Shamir (2011). Optimal multiyear management of a water supply 646 system under uncertainty: Robust counterpart approach, Water Resour. Res., 47(10), W10515, doi: $647 \quad 10.1029 / 2011 w r 010596$.

648 Jayaram, N. and K. Srinivasan (2008). Performance-based optimal design and rehabilitation of water 649 distribution networks using life cycle costing, Water Resources Research, 44(1), W01417, doi: $650 \quad 10.1029 / 2006$ WR005316.

651 Kang, D. S., M. F. K. Pasha and K. Lansey (2009). Approximate methods for uncertainty analysis of 652 water distribution systems, Urban Water Journal, 6(3), 233-249, 10.1080/15730620802566844. 
Kapelan, Z. S., D. A. Savic and G. A. Walters (2005). Multiobjective design of water distribution

654 systems under uncertainty, Water Resources Research, 41(11), W11407, doi:10.1029/2004WR003787.

656 Liu, H., D. Savić, Z. Kapelan, M. Zhao, Y. Yuan and H. Zhao (2014). A diameter-sensitive flow 657 entropy method for reliability consideration in water distribution system design, Water Resources Research, 50(7), 5597-5610, doi: 10.1002/2013WR014882.

659 Maier, H. R., B. J. Lence, B. A. Tolson and R. O. Foschi (2001). First-order reliability method for 660 estimating reliability, vulnerability, and resilience, Water Resources Research, 37(3), 779-790, doi: 10.1029/2000WR900329.

662 Marchi, A., E. Salomons, A. Ostfeld, Z. Kapelan, A. Simpson, A. Zecchin, H. Maier, Z. Wu, S. 663 Elsayed, Y. Song, T. Walski, C. Stokes, W. Wu, G. Dandy, S. Alvisi, E. Creaco, M. Franchini, J. 664 Saldarriaga, D. Páez, D. Hernández, J. Bohórquez, R. Bent, C. Coffrin, D. Judi, T. McPherson, P. van 665 Hentenryck, J. Matos, A. Monteiro, N. Matias, D. Yoo, H. Lee, J. Kim, P. Iglesias-Rey, F. 666 Martínez-Solano, D. Mora-Meliá, J. Ribelles-Aguilar, M. Guidolin, G. Fu, P. Reed, Q. Wang, H. Liu, 667 K. McClymont, M. Johns, E. Keedwell, V. Kandiah, M. Jasper, K. Drake, E. Shafiee, M. Barandouzi, 668 A. Berglund, D. Brill, G. Mahinthakumar, R. Ranjithan, E. Zechman, M. Morley, C. Tricarico, G. de 669 Marinis, B. Tolson, A. Khedr and M. Asadzadeh (2014). Battle of the Water Networks II, Journal of 670 Water Resources Planning and Management, 140(7), 04014009, 671 doi:10.1061/(ASCE)WR.1943-5452.0000378.

672 Martinez, J. (2010). Cost and reliability comparison between branched and looped water supply 673 networks, Journal of Hydroinformatics, 12(2), 150-160. 
674 Morley, M. S. and C. Tricarico (2008). Pressure driven demand extension for EPANET 675 (EPANETpdd). Technical Report 2008-02. Exeter, UK, Centre for Water System, University of 676 Exeter.

677 Ostfeld, A. (2004). Reliability analysis of water distribution systems, Journal of Hydroinformatics, 678 6(2004), 281-294.

679 Ostfeld, A. (2012). Optimal reliable design and operation of water distribution systems through 680 decomposition, Water Resources Research, 48(10), W10521, doi:10.1029/2011wr011651.

681 Park, J. I., J. H. Lambert and Y. Y. Haimes (1998). Hydraulic power capacity of water distribution 682 networks in uncertain conditions of deterioration, Water Resources Research, 34(12), 3605-3614, doi: $68310.1029 / 98 w r 01377$.

684 Piratla, K. R. and S. T. Ariaratnam (2012). Reliability based optimal design of water distribution 685 networks considering life cycle components, Urban Water Journal, 9(5), 305-316, $68610.1080 / 1573062 x .2012 .660961$.

687 Prasad, T. and N. Park (2004). Multiobjective genetic algorithms for design of water distribution 688 networks, Journal of Water Resources Planning and Management, 130(1), 73-82, 689 doi:10.1061/(ASCE)0733-9496(2004)130:1(73).

690 Raad, D. N., A. N. Sinske and J. H. van Vuuren (2010). Comparison of four reliability surrogate 691 measures for water distribution systems design, Water Resour. Res., 46(5), W05524, doi: $69210.1029 / 2009 w r 007785$.

693 Rossman, L. A. (2000). EPANET 2: users manual. US, EPA. 
694 Savic, D. A. and G. A. Walters (1997). Genetic algorithms for least-cost design of water distribution 695 networks, Journal of Water Resources Planning and Management, 123(2), 67-77, doi: 696 10.1061/(ASCE)0733-9496(1997)123:2(67).

697 Shafiqul Islam, M., R. Sadiq, M. Rodriguez, H. Najjaran and M. Hoorfar (2014). Reliability 698 Assessment for Water Supply Systems under Uncertainties, Journal of Water Resources Planning and 699 Management, 140(4), 468-479, doi:10.1061/(ASCE)WR.1943-5452.0000349.

700 Sumer, D. and K. Lansey (2009). Effect of uncertainty on water distribution system model design 701 decisions, Journal of Water Resources Planning and Management, 135(1), 38-47, doi: 702 10.1061/(ASCE)0733-9496(2009)135:1(38).

Sun, S., S. T. Khu, Z. Kapelan and S. DjordjeviC (2011). A fast approach for multiobjective design of water distribution networks under demand uncertainty, Journal of Hydroinformatics, 13(2), 143-152. Tanyimboh, T. T. and A. B. Templeman (2000). A quantified assessment of the relationship 706 between the reliability and entropy of water ditribution systems, Engineering Optimization, 33(2), 179-199, doi:10.1080/03052150008940916.

Todini, E. (2000). Looped water distribution networks design using a resilience index based heuristic approach, Urban Water, 2(2), 115-122, doi: 10.1016/S1462-0758(00)00049-2.

710 Torii, A. and R. Lopez (2012). Reliability Analysis of Water Distribution Networks Using the 711 Adaptive Response Surface Approach, Journal of hydraulic engineering, 138(3), 227-236, 712 doi:10.1061/(ASCE)HY.1943-7900.0000504.

713 van Zyl, J., O. Piller and Y. le Gat (2008). Sizing Municipal Storage Tanks Based on Reliability 714 Criteria, Journal of Water Resources Planning and Management, 134(6), 548-555, doi:10.1061/(ASCE)0733-9496(2008)134:6(548). 
716 Walski, T. (2001). The Wrong Paradigm-Why Water Distribution Optimization Doesn't Work,

717 Journal of Water Resources Planning and Management, 127(4), 203-205,

718 doi:10.1061/(ASCE)0733-9496(2001)127:4(203).

719 Walski, T. M. (1993). Water distribution valve topology for reliability analysis, Reliability

720 Engineering \& System Safety, 42(1), 21-27, doi:org/10.1016/0951-8320(93)90051-Y.

721 Wang, Q., M. Guidolin, D. Savic and Z. Kapelan (2014). Two-Objective Design of Benchmark

722 Problems of a Water Distribution System via MOEAs: Towards the Best-Known Approximation of 723 the True Pareto Front, Journal of Water Resources Planning and Management, 141(3), 04014060,

724 10.1061/(ASCE)WR.1943-5452.0000460.

725 Xu, C. and I. C. Goulter (1999). Reliability-based optimal design of water distribution networks, 726 Journal of Water Resources Planning and Management, 125(6), 352-362, 727 doi:10.1061/(ASCE)0733-9496(1999)125:6(352)

728 Yannopoulos, S. and M. Spiliotis (2013). Water distribution system reliability based on minimum

729 cut-set approach and the hydraulic availability, Water Resources Management, 27(6), 1821-1836, 730 doi:10.1007/s11269-012-0163-5.

731 Zhuang, B., K. Lansey and D. Kang (2013). Resilience/Availability Analysis of Municipal Water

732 Distribution System Incorporating Adaptive Pump Operation, Journal of hydraulic engineering, 733 139(5), 527-537, doi:10.1061/(ASCE)HY.1943-7900.0000676. 
Table 1. List of Abbreviations

\begin{tabular}{ll}
\hline API & Available power index \\
DSFE & Diameter-sensitive flow entropy \\
FFD & Fraction of failure degree \\
FNC & Failure node count \\
RI & Resilience index \\
HGL & Hydraulic grade line \\
LHS & Latin hypercube sampling \\
MRI & Modified resilience index \\
NRI & Network resilience index \\
PDD & Pressure driven demand \\
PDF & Probability density function \\
PFS & Percentage of failure scenario \\
PHRI & Pipe hydraulic resilience index \\
WDS & Water distribution system \\
\hline
\end{tabular}



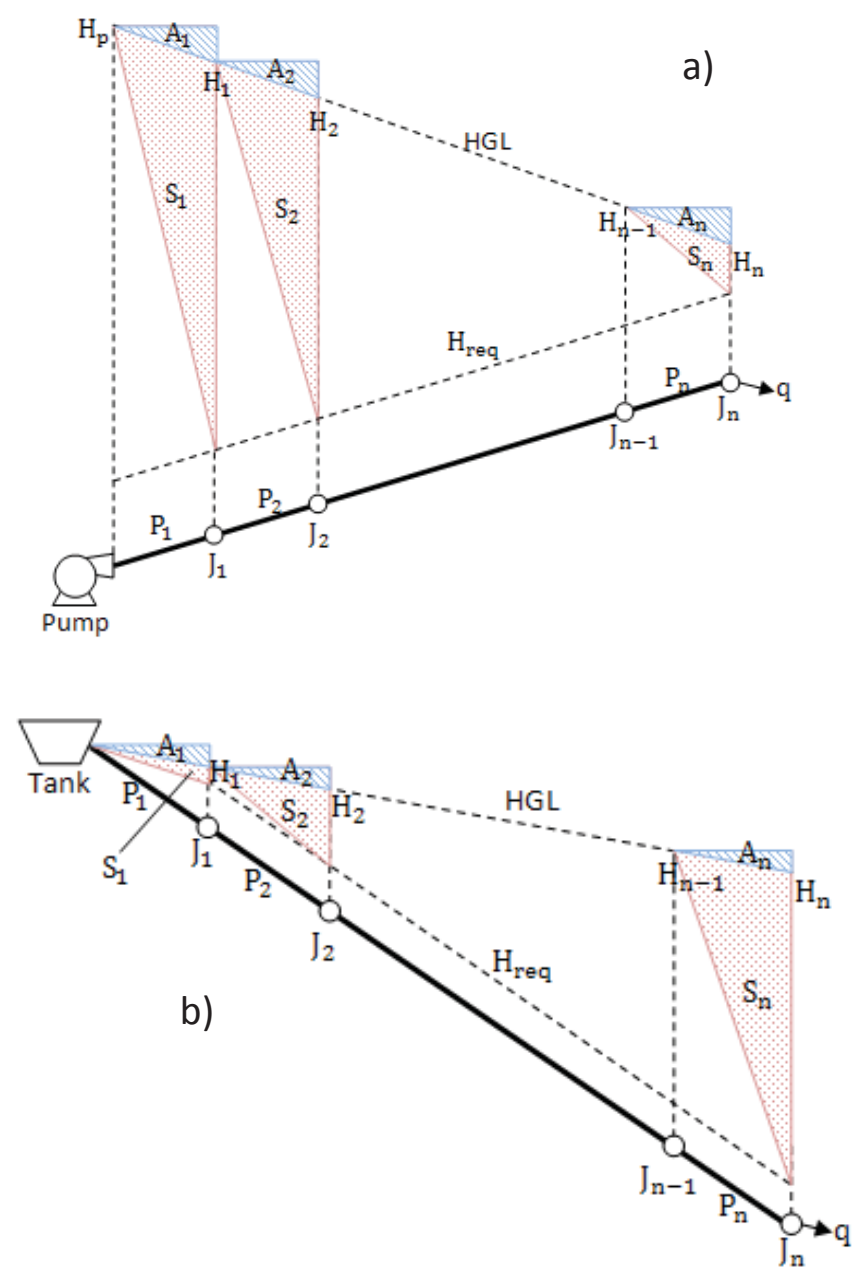

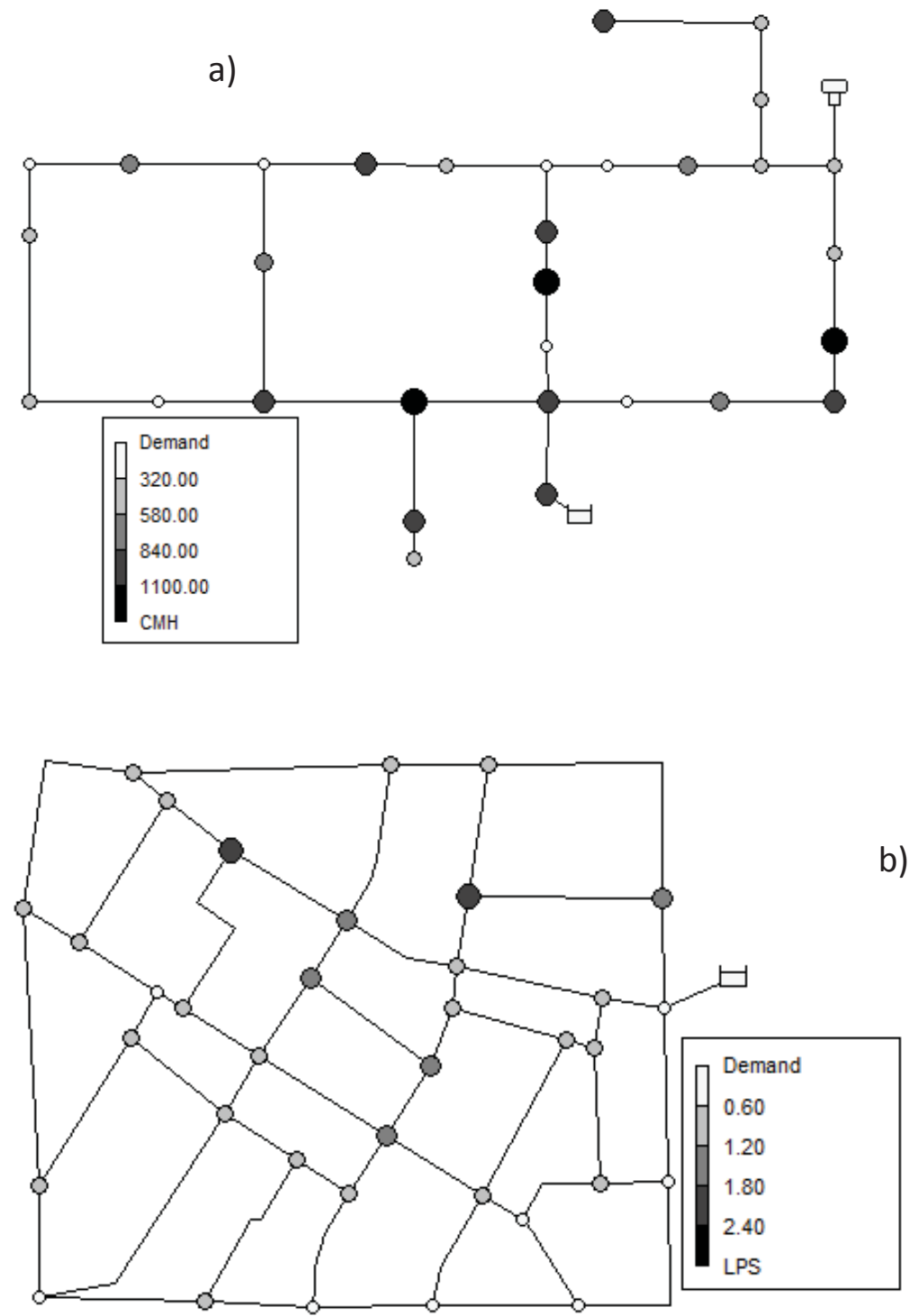

b) 


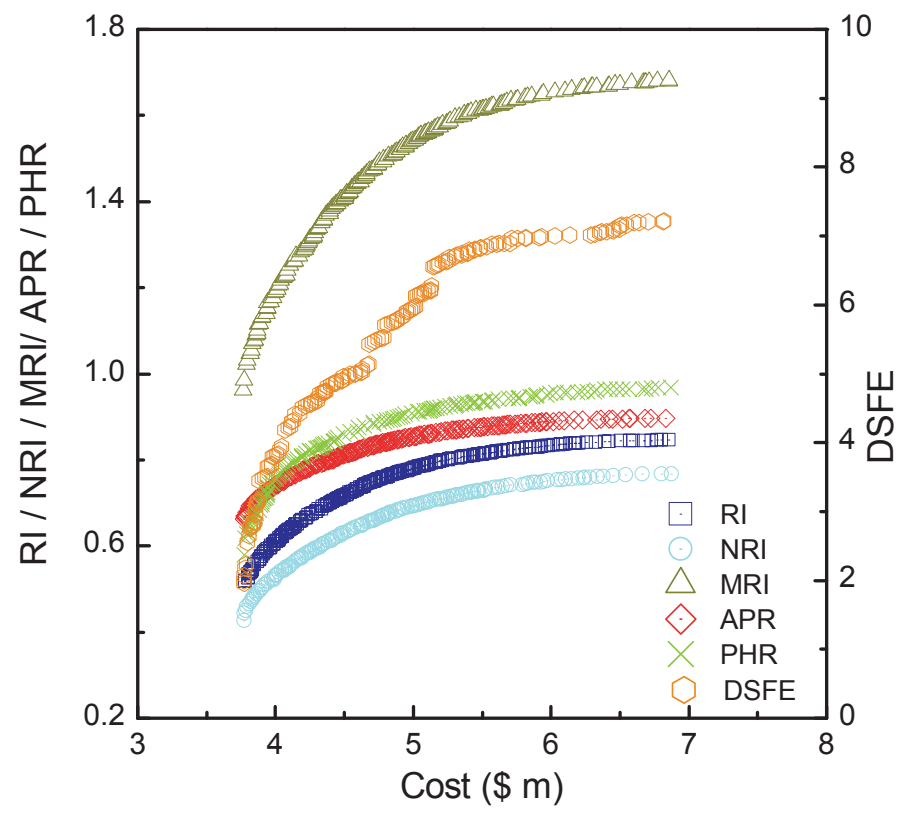




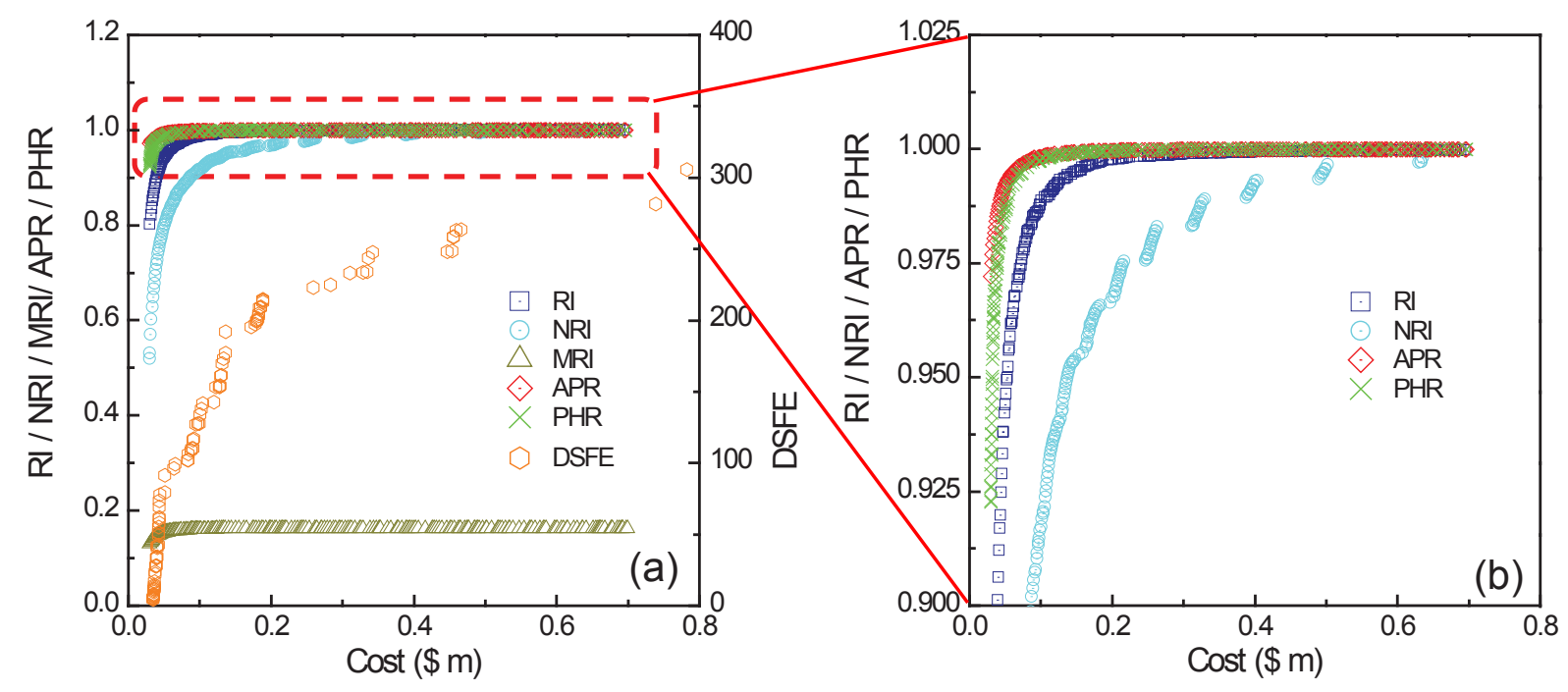



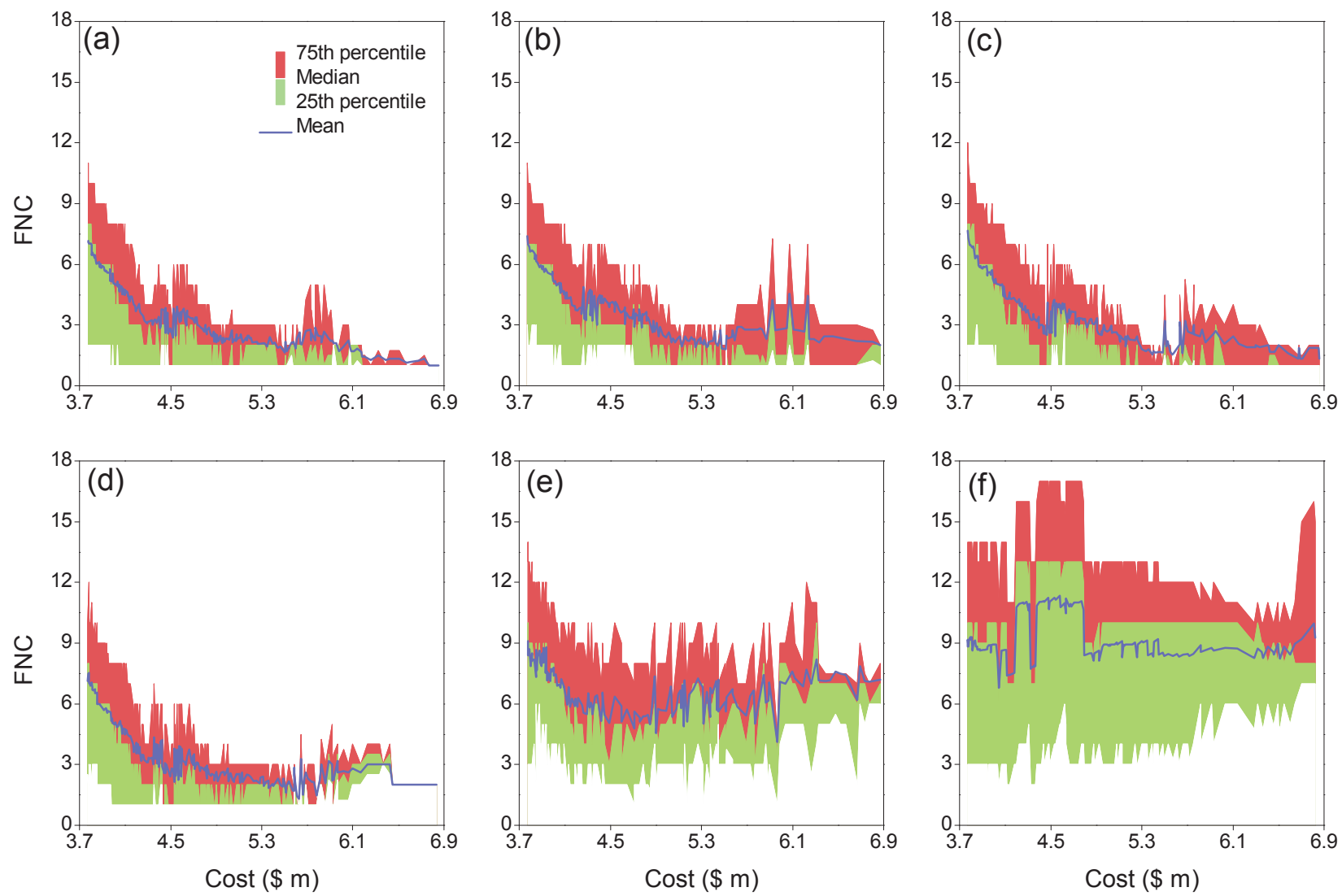

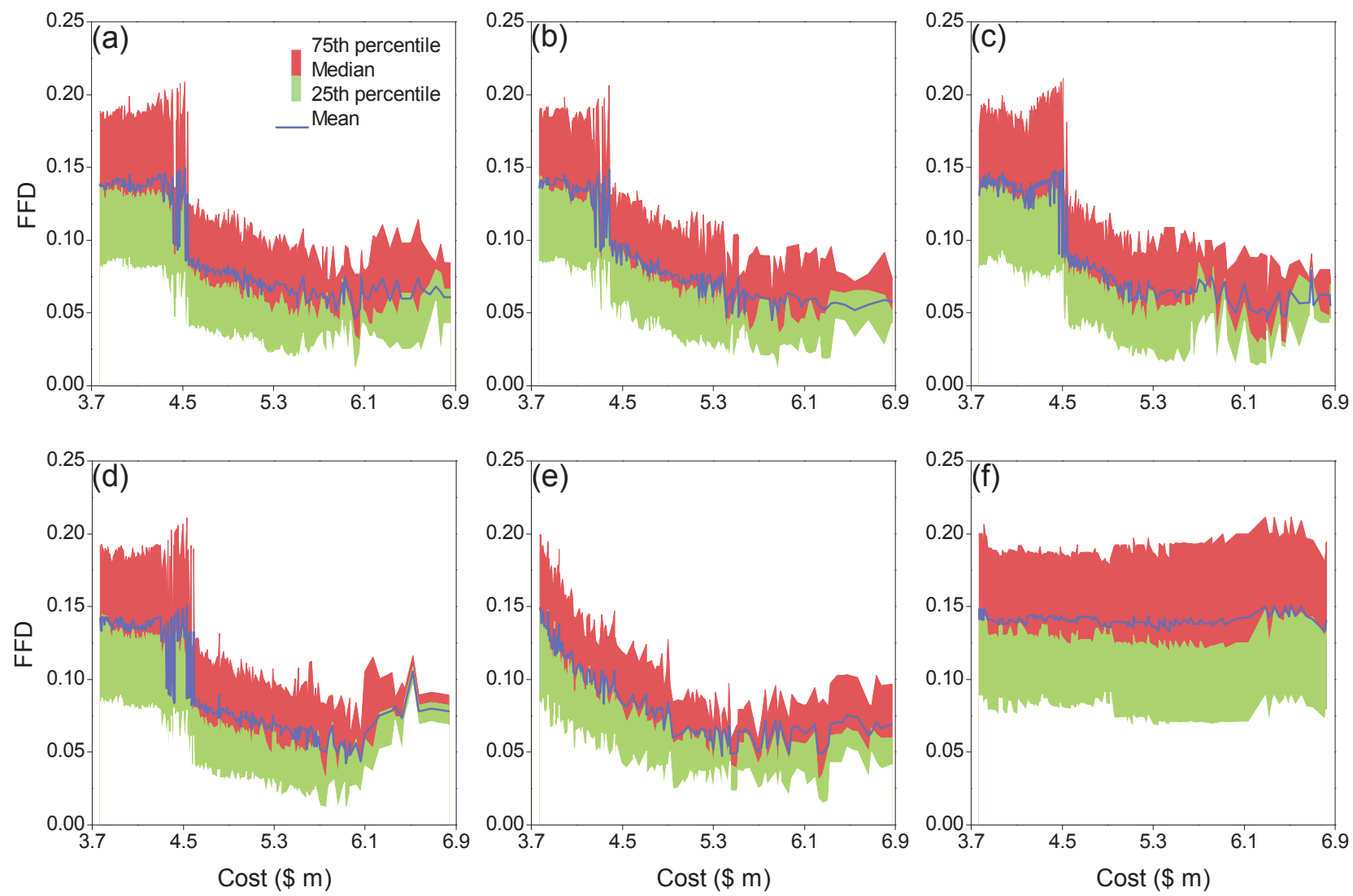

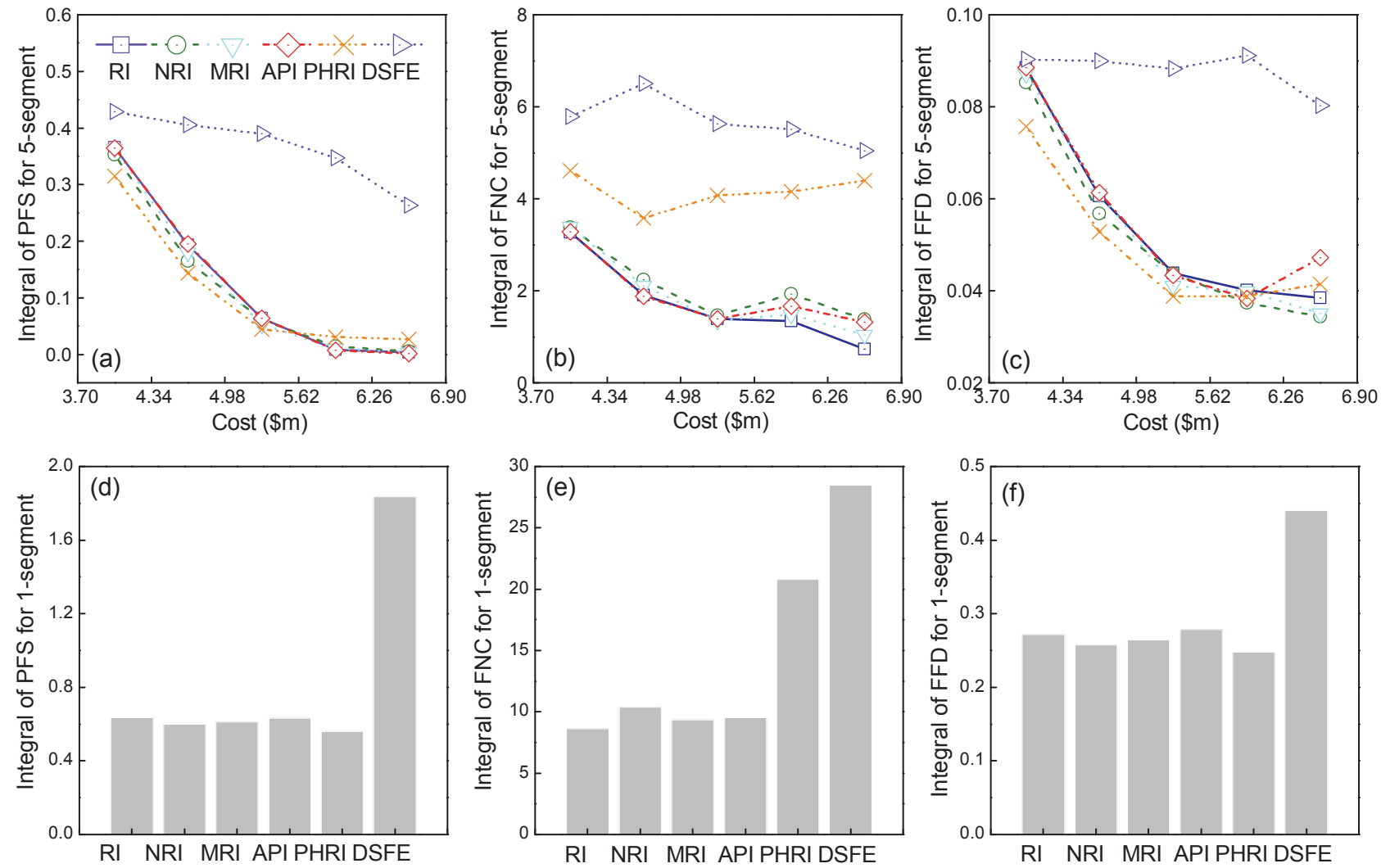

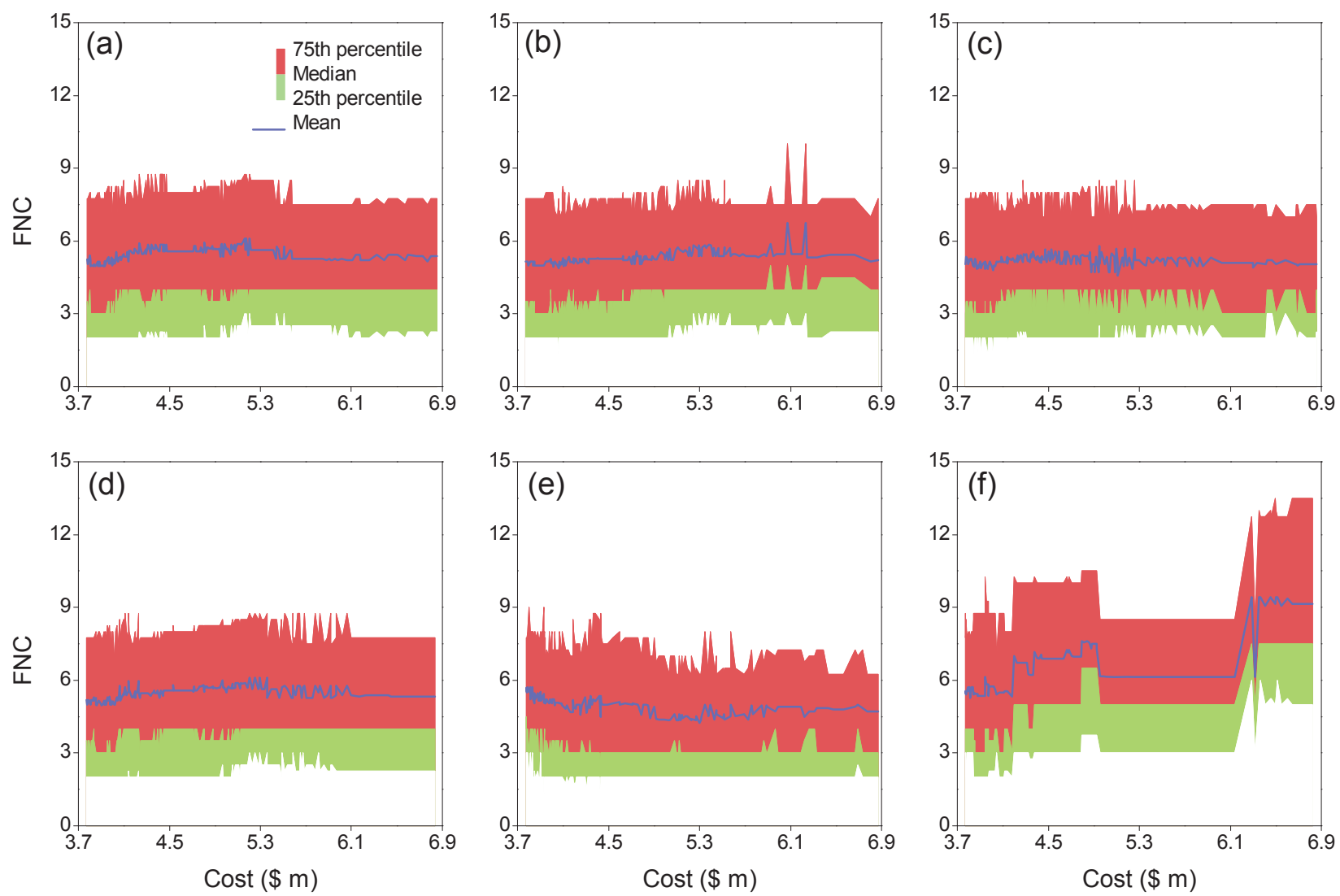

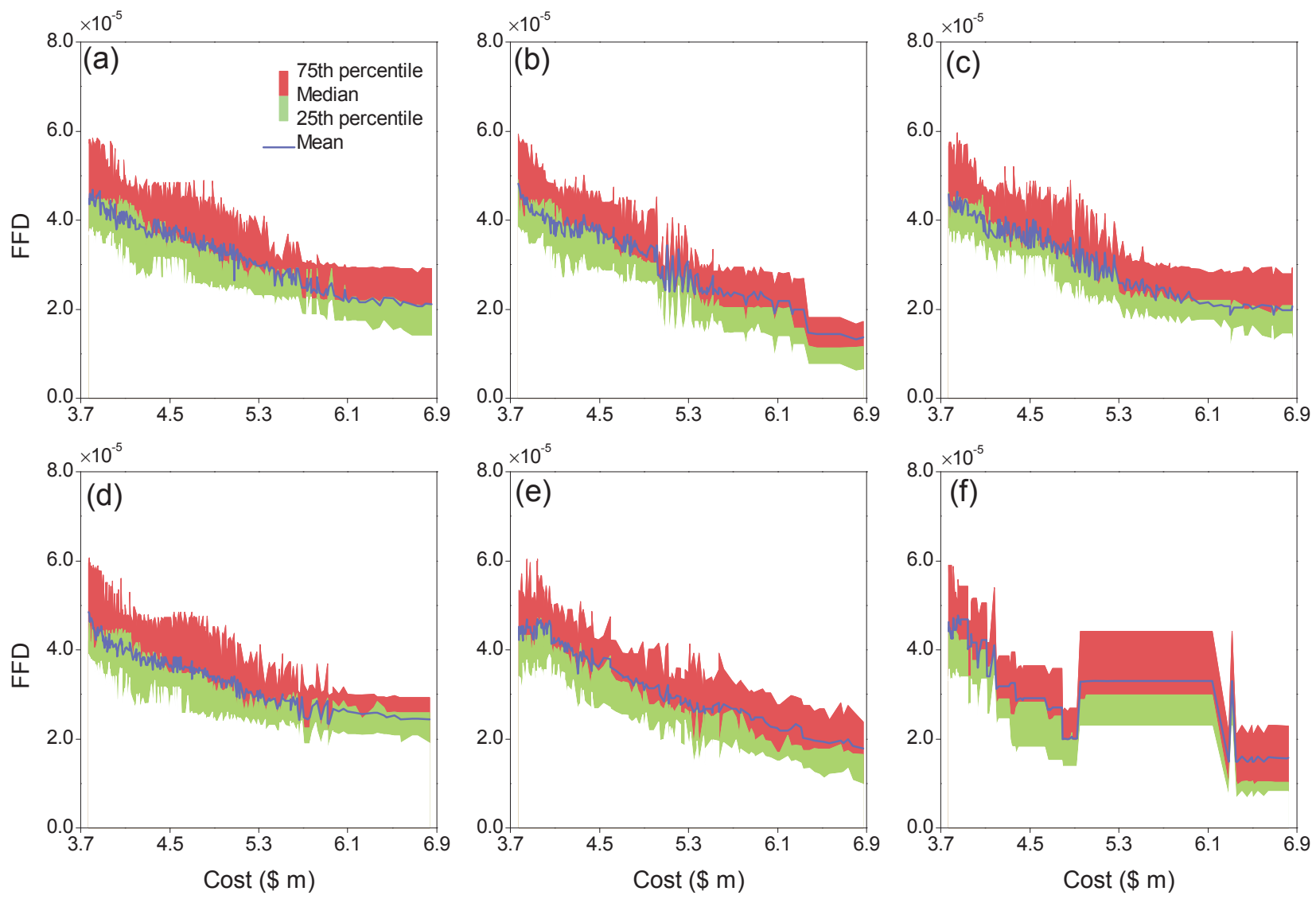

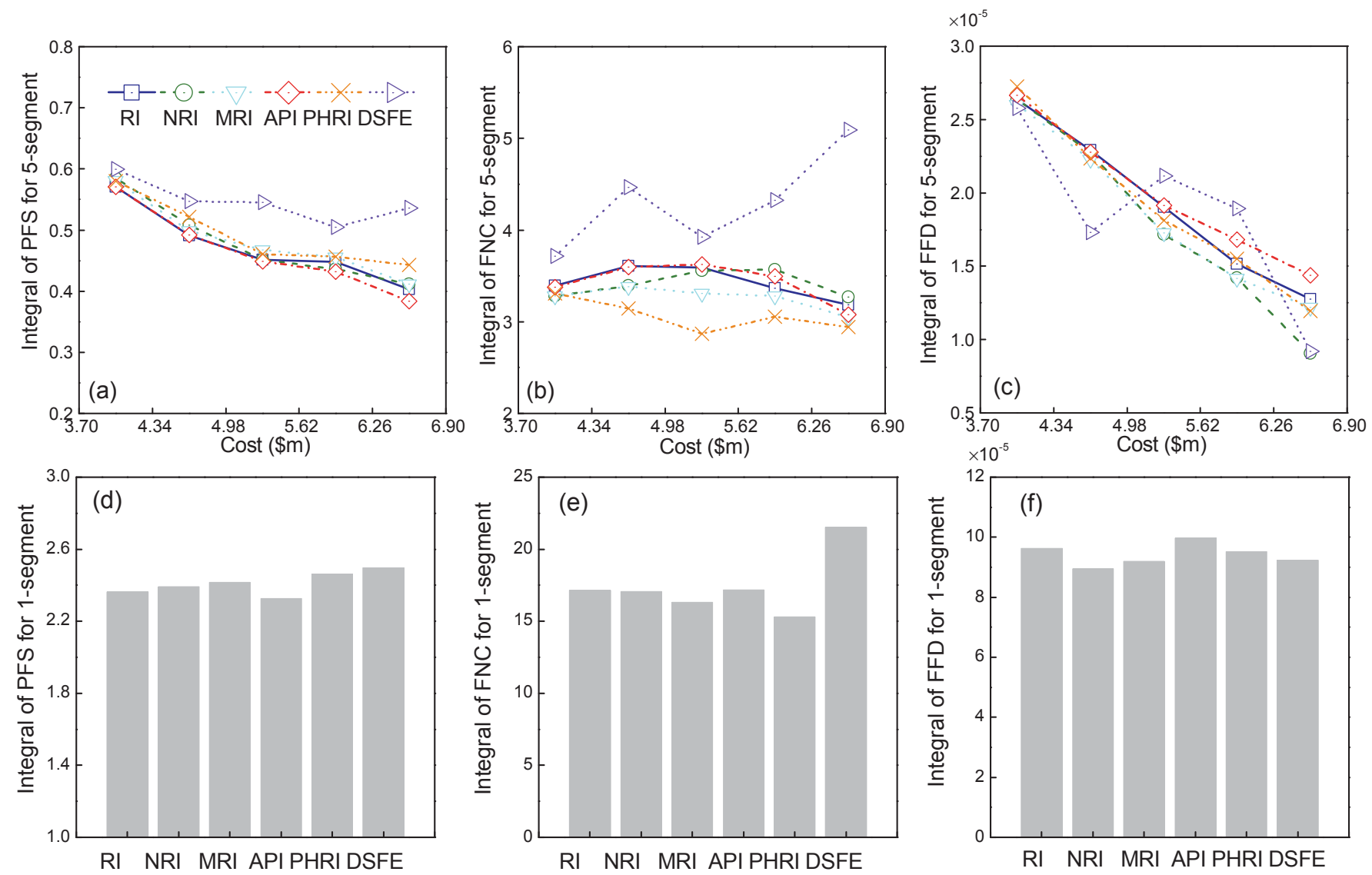

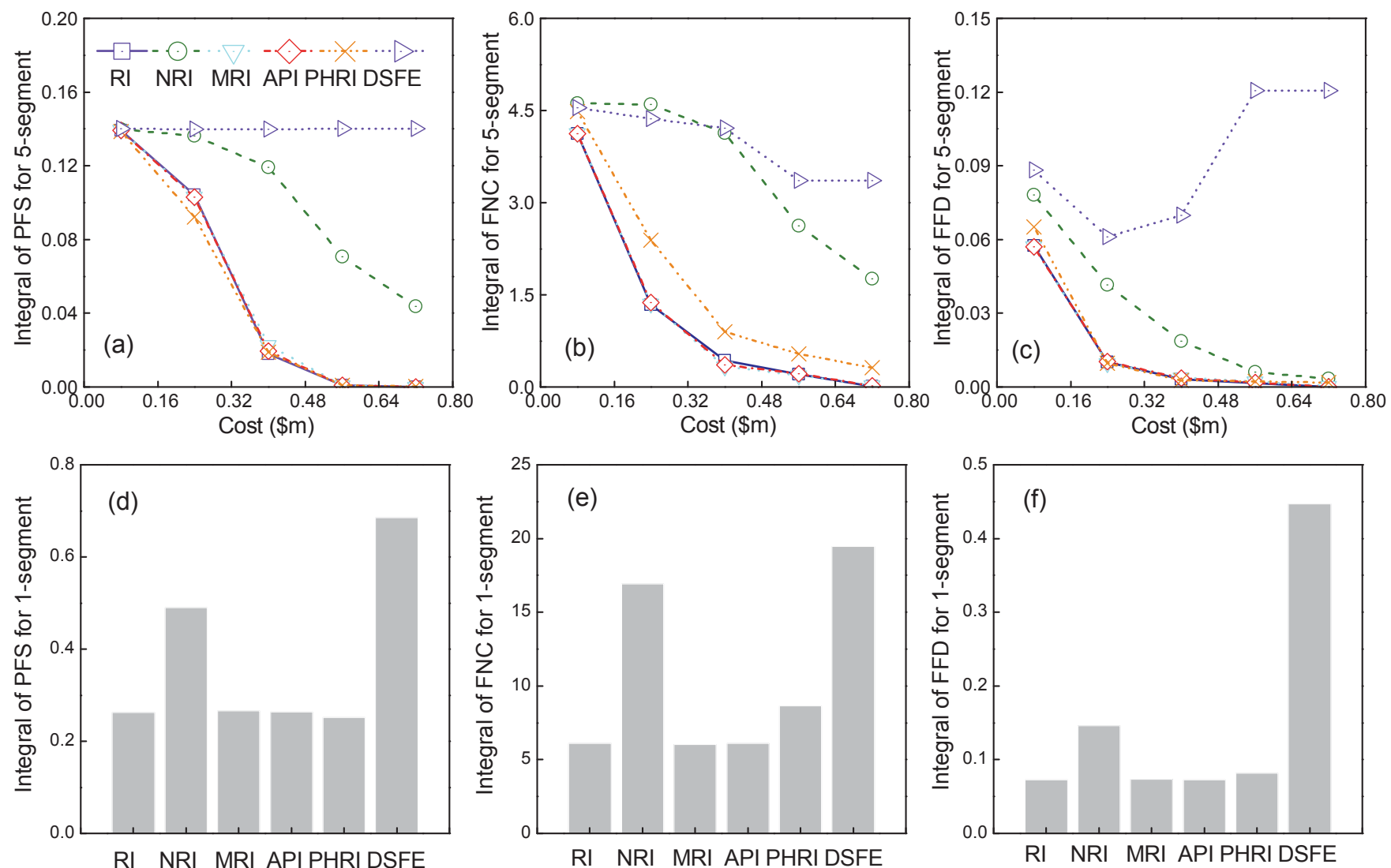

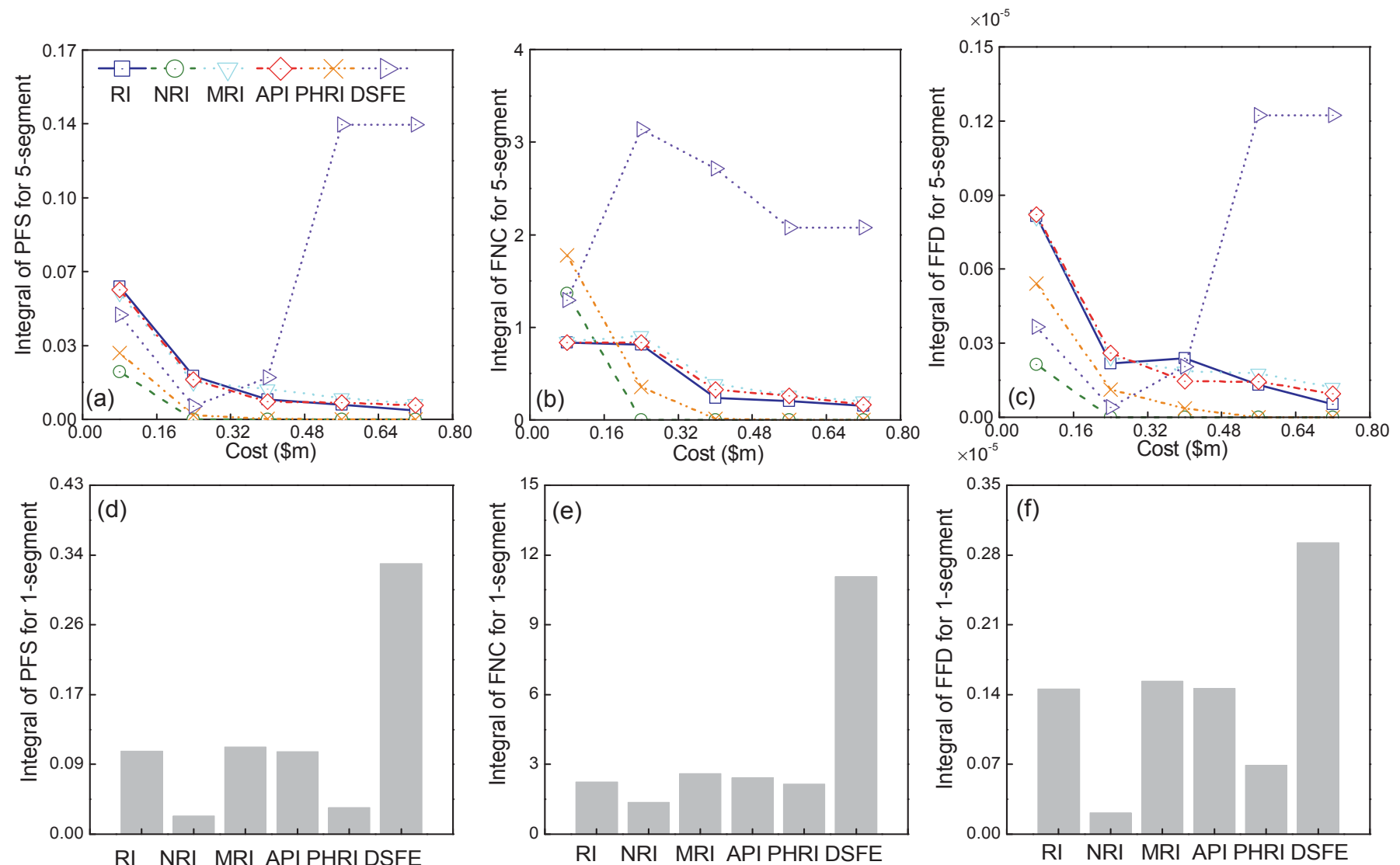


\section{Figure captions}

Figure 1. A diagram of water distribution systems with HGL (a) a pressurized supply system, (b) a gravity supply system. The two systems are assumed with only a fixed demand $q$ at node $J_{n}$

Figure 2. The schematic diagram with nodal demand represented by the bubble size: (a) Hanoi network; (b) Fossolo network

Figure 3. Pareto fronts obtained using each reliability surrogate measure and cost objectives for the Hanoi network

Figure 4. Pareto fronts obtained using each reliability surrogate measure and cost objectives for the Fossolo network: (a) Pareto fronts derived by six surrogate reliability measures; (b) enlarged part of Figure 4a

Figure 5. Failure node count under perturbation of demand uncertainty in the Hanoi network: (a) RI, (b) NRI, (c) MRI, (d) API, (e) PHRI, and (f) DSFE

Figure 6. Fraction of failure degree under perturbation of demand uncertainty in the Hanoi network: (a) RI, (b) NRI, (c) MRI, (d) API, (e) PHRI, and (f) DSFE

Figure 7. Integral of evaluation indicators under perturbation of demand uncertainty in the Hanoi network. PFS is the percentage of failure scenarios with both integral approaches (Figures 7a and 7d); FNC is the failure node count with both integral approaches (Figures $7 \mathrm{~b}$ and 7e); and FFD is the fraction of failure degree with both integral approaches (Figures $7 \mathrm{c}$ and $7 \mathrm{f}$ ).

Figure 8. Failure node count under perturbation of pipe failure in the Hanoi network: (a) RI, (b) NRI, (c) MRI, (d) API, (e) PHRI, and (f) DSFE

Figure 9. Fraction of failure degree under disturbance of pipe failure uncertainty in the Hanoi network: (a) RI, (b) NRI, (c) MRI, (d) API, (e) PHRI, and (f) DSFE

Figure 10. Integral of evaluation indicators under perturbation of pipe failure uncertainty in the Hanoi network. PFS is the percentage of failure scenarios with both integral approaches (Figures 10a and 10d); FNC is the failure node count with both integral approaches (Figures 10b and 10e); and FFD is the fraction of failure degree with both integral approaches(Figures 10c and 10f) 
Figure 11. Integral of evaluation indicators under perturbation of demand uncertainty in the Fossolo network. PFS is the percentage of failure scenarios with both integral approaches (Figures 11a and 11d); FNC is the failure node count with both integral approaches (Figures 11b and 11e); and FFD is the fraction of failure degree with both integral approaches (Figures 11c and 11f)

Fiugre 12. Integral of evaluation indicators under perturbation of pipe failure uncertainty in the Fossolo network. PFS is the percentage of failure scenarios with both integral approaches (Figures 12a and 12d); FNC is the failure node count with both integral approaches (Figures 12b and 12e); and FFD is the fraction of failure degree with both integral approaches (Figures 12c and 12f) 